\title{
Probing low energy and mass scales
}

\author{
Oliviero Cremonesi ${ }^{\mathrm{a}}$ and Alessandro Melchiorri ${ }^{\mathrm{b}}$ \\ aINFN Sez. di Milano Bicocca and Dipartimento di Fisica "G.Occhialini", Universitá degli studi di \\ Milano Bicocca, Piazza della Scienza 3, I-20126 Milano,Italy \\ ${ }^{\text {b}}$ Dipartimento di Fisica and Sezione INFN, Università degli Studi di Roma "La Sapienza", P.le Aldo \\ Moro 5, 00185, Rome, Italy
}

Low energy neutrino processes are ideal probes for new Physics beyond the Standard Model. Cosmological observations and rare nuclear processes can test neutrino mass scales and give definite answers to unsolved basic questions like the Majorana/Dirac nature of the neutrino.

\section{Introduction}

Well proved by a number of experiments carried out during the last ten years, the existence of neutrino oscillations has stimulated renewed interest in the study of neutrino properties. Neutrino oscillation results have shown that neutrinos mix and have finite masses; they have thus provided us with the first clear evidence of phenomena beyond the reach of Standard Model (SM). Unlike quark mixings, neutrino mixings are large, although the reason for this is not yet fully understood. On the other hand neutrino masses are much smaller than those of the charged leptons and their pattern (or absolute scale) is still unknown. Neutrino oscillations in fact only depend on the absolute value of the difference of the squares of the neutrino masses and two possible hierarchies (or orderings) are then implied by current available data: the normal $\left(m_{1}<m_{2} \ll m_{3}\right)$ and the inverted hierarchy $\left(m_{3} \ll m_{1}<m_{2}\right)$. These and other unsolved questions concerning neutrino properties are becoming subject of increasing interest and considered a unique tool to see what new Physics lies beyond SM predictions. This holds in particular for the Dirac/Majorana neutrino nature which represents one of the most important open questions in neutrino Physics. In the SM neutrinos are Dirac particles by construction (i.e. in order to conserve lepton number L). In the limit of vanishing masses however Lepton Number conservation can be equivalently stated in terms of neutrino helicity properties and the Majorana or Dirac descriptions for neutrino are equivalent (i.e. don't change the physical content of the theory). For finite neutrino masses however the two descriptions are no more equivalent and can give rise to different Physical scenarios (e.g. mass generation mechanisms).

Only experiments sensitive to $\mathrm{m}_{1}$ can aim at solving the mass hierarchy problem. This is the case for kinematic measurements of the $\beta$ spectrum end-point, neutrinoless double beta decay $(\beta \beta[0 \nu])$ and cosmological measurements. Intense efforts are therefore being spended to fix (measure or bound) the absolute scale of neutrino masses (as contrasted to the $\Delta m^{2}$ measurements of neutrino oscillation experiments) with various observations and experiments, characterized by different systematics and sensitivities. Cosmological observations and laboratory experiments actually probe different quantities which can be however compared within specific models. In the case of 3 Majorana neutrinos and upon a good control of the systematics, cosmological observations and $\beta \beta[0 \nu]$ experiments have the largest success potential. In the same theoretical framework, complementary informations can come also from oscillation experiments if $\mathrm{U}_{e 3}$ is large enough. Kinematical measurements seem presently characterized by a lower sensitivity although they represent the only model independent measurement of the neutrino mass scale. On the other hand $\beta \beta[0 \nu]$ experiments 
are the only presently available tool to probe the Dirac/Majorana nature of the neutrino.

It should be stressed that the 3 Majorana neutrino model is the most likely but not the unique framework for the measurements discussed above. Different extensions of the SM could equally account for such observations. Moreover there is no possibility to account for the LSND result within the the 3 Majorana neutrino model.

The present status and future perspectives of the esperimental (cosmological observations and laboratory measurements) and theoretical efforts in the framework of neutrino masses were discussed in dedicated talks of the present session and in variuos talks of the general sessions. A general overview from the point of view of terrestrial and sky observations will be given in the next sections. A possible scenario for future developments in the forthcoming years will be summarized in the last section.

\section{Laboratory measurements}

Neutrino absolute mass scale and nature are two of the most relevant open questions in neutrino Physics addressed by next generation laboratory experiments. All proposed experiments are based on the precise study of proper nuclear processes: single beta decay for the direct measurement of the electron antineutrino mass and neutrinoless double beta decay as a unique probe of the basic neutrino properties.

\subsection{Neutrinoless $\beta \beta$ decay}

Having no conserved charge except for the Lepton number L, neutrinos are the only truly "neutral" fermions characterized by the possibility of being their own anti-particles. If, as predicted in many extensions of the SM, L is in fact not conserved, neutrinos could be the unique fermions with a Majorana nature. The implications of Majorana or Dirac massive neutrinos are completely different and the question of their nature is therefore an essential building block for every possible extension of the Standard Model. The only practical method to attack this problem is the study of neutrinoless double beta decay [1, a rare nuclear process in which a nucleus (A,
$\mathrm{Z})$ would turn into an isobar $(\mathrm{A}, \mathrm{Z}+2)$ by transforming two neutrons into protons while emitting two electrons: $(\mathrm{A}, \mathrm{Z}) \rightarrow(\mathrm{A}, \mathrm{Z}+2)+2 \mathrm{e}^{-}$ - This differs from "normal" double-beta decay or $\beta \beta[2 \nu]$ (second order process of the weak interaction), which is rare but has been detected: $(\mathrm{A}, \mathrm{Z}) \rightarrow(\mathrm{A}, \mathrm{Z}+2)+2 \mathrm{e}^{-}+2 \nu . \beta \beta[0 \nu]$ can occur through different processes but all of them require that the neutrino is a Majorana particle. Moreover, when induced by the exchange of a massive Majorana neutrino, the $\beta \beta[0 \nu]$ width is proportional to an effective parameter (neutrino mass) $\left|\left\langle m_{e e}\right\rangle\right|=\Sigma_{k} U_{e k}^{2} m_{k}$ through the phase space factor $\mathrm{G}^{0 \nu}$ and nuclear matrix element $\mathrm{M}^{0 \nu}$

$(\tau)^{-1}=G^{0 \nu}\left|M^{0 \nu}\right|^{2}\left\langle m_{\nu}\right\rangle^{2} / m_{e}^{2}$

$\beta \beta[0 \nu]$ observation would allow to obtain therefore relevant informations on the neutrino absolute mass scale. Large uncertainties in the nuclear matrix elements calculations imply serious difficulties in the comparison between experiments on different $\beta \beta[0 \nu]$ active isotopes and represent the most relevant source of systematics in the evaluation of $\left|\left\langle m_{e e}\right\rangle\right|$. The subject was discussed in the talk of Fedor Simkovic who described a new calculation method, based on the normalization of the relevant QRPA parameters to the $\beta \beta[2 \nu]$ results, characterized by a better stability with respect to previous QRPA calculations. $\beta \beta[2 \nu]$ results are unfortunately not available for all $\beta \beta[0 \nu]$ active isotopes and the method is not yet fully accepted by the whole community involved in $\mathrm{M}^{0 \nu}$ calculations. More efforts in this field are therefore strongly encouraged and experimental analyses on different active $\beta \beta[0 \nu]$ isotopes are required.

Besides the general motivations discussed above, $\beta \beta[0 \nu]$ is presently a hot and debated subject for two more reasons: i) the claim for a positive effect in the Heidelberg-Moscow data on ${ }^{76} \mathrm{Ge}$; ii) in the inverted hierarchy scenario, present neutrino oscillation results suggest a $\left|\left\langle m_{e e}\right\rangle\right|$ value of the order of $50 \mathrm{meV}$, in the reach of next generation experiments.

Two presently running $\beta \beta[0 \nu]$ experiments have the potential to confirm the ${ }^{76} \mathrm{Ge}$ claim and probe the inverted hierarchy region of neutrino masses: CUORICINO $[3]$ at LNGS and NEMO3 [4] in the Frejus tunnel. 
Based on the bolometric technique, CUORICINO exploits a fully calorimetric approach and was introduced in the talk of Maura Pavan. A limit of $2.4 \times 10^{24} \mathrm{y}$ on the half-lifetime for $\beta \beta[0 \nu]$ of ${ }^{130} \mathrm{Te}$ was presented, corresponding to a limit on the effective neutrino mass in the range 0.18 $1.05 \mathrm{eV}$. With a total mass of nearly $41 \mathrm{~kg}$ of natural $\mathrm{TeO}_{2}$ and a satisfactory duty cycle, CUORICINO is running since 2003 in Hall A of the Gran Sasso underground Laboratory. The achieved background level is $0.18 \pm 0.01 \mathrm{c} / \mathrm{keV} / \mathrm{kg} / \mathrm{y}$ corresponding to a $5 \mathrm{y}$ sensitivity of $8.7 \times 10^{24} \mathrm{y}$ on the ${ }^{130} \mathrm{Te} \beta \beta[0 \nu]$ half-lifetime.

Characterized by a similar sensitivity but a completely different approach, NEMO3 was discussed by Ladislav Vala. Advantaged by a well known technology, this tracking + calorimetric detector can study different nuclei simultaneously and identify the $\beta \beta$ events by reconstructing the electron tracks. NEMO3 is installed in the Modane underground laboratory and has produced excellent results on the $\beta \beta[2 \nu]$ decay of various nuclei: ${ }^{100} \mathrm{Mo},{ }^{82} \mathrm{Se},{ }^{150} \mathrm{Nd},{ }^{116} \mathrm{Cd}$ and ${ }^{96} \mathrm{Zr}$. An upper limit of $5.8 \times 10^{23} \mathrm{y}$ on the half-lifetime for $\beta \beta[0 \nu]$ of ${ }^{100} \mathrm{Mo}$ was presented, corresponding to a limit on the effective neutrino mass in the range $0.6-0.9 \mathrm{eV}$. The projected $5 \mathrm{y}$ sensitivity on ${ }^{100} \mathrm{Mo} \beta \beta[0 \nu]$ halflife is $4.0 \times 10^{23}$ y corresponding to a $\left|\left\langle m_{e e}\right\rangle\right| 0.2-0.35$ range. Both CUORICINO and NEMO3 have the sensitivity to confirm the ${ }^{76} \mathrm{Ge}$ claim. A disproof is however impossible for both of them because of the large uncertainties in the nuclear matrix elements calculations. Different would be the case for ${ }^{76} \mathrm{Ge}$ experiments for which a direct comparison would be possible 8 . A defnite answer to the ${ }^{76}$ Ge claim should be in any case possible with the upcoming next generation experiments, characterized by improved masses ( $\sim 1$ ton) and background levels (1-10 counts/keV/ton/y). Main goal of these experiments is to probe the inverted hierarchy of the Majorana neutrino mass scale. Sensitivities better than $\sim 50 \mathrm{meV}$ on $\left|\left\langle m_{e e}\right\rangle\right|$ are therefore foreseen. In most cases they are extensions of previous experiments exploiting the most successful techniques. Variuos 1 ton experiments have been so far proposed and some of them are already in construction. A general introduction to the
USA efforts (mainly Majorana 5 on ${ }^{76} \mathrm{Ge}$ and EXO 6] on ${ }^{136} \mathrm{Xe}$ ) was given by Frank Avignone. The bolometric CUORE [7] experiment on ${ }^{130} \mathrm{Te}$, of which CUORICINO is a successful demonstrator, is in construction at LNGS. Its completion is foreseen within the end of 2010 and was presented by Maura Pavan. Alessandro Bettini introduced GERDA [8, also in construction at LNGS, whose first phase (with just $20 \mathrm{~kg}$ of isotopically enriched Ge) would allow to definitely test the ${ }^{76} \mathrm{Ge}$ claim within 2010. Finally, SUPERNEMO [9] (extention of NEMO3) was disussed by V. Lala.

\subsection{Single $\beta$ decay}

The precise measurement of the electron spectrum in $\beta$-decays is the only laboratory technique for the direct measurement of a small neutrino mass, without additional assumptions on the character of the neutrino. The neutrino mass (or an upper limit to it) is inferred from the shape of the energy spectrum near its kinematical end point. The shape of the spectrum near the kinematical end point depends on the masses of all three mass states and the measurement is sensitive to an effective mass $\mathrm{m}_{\beta}=\sqrt{\Sigma_{k}\left|U_{e k}\right|^{2} m_{k}^{2}}$. The relative number of events occurring in an interval of kinetic energy $\Delta \mathrm{T}$ near the end-point is proportional to $(\Delta \mathrm{T} / \mathrm{Q})^{3}$ so that very low $\mathrm{Q}$-values are required. Even exploiting the lowest $\mathrm{Q}$-value $\beta$ processes available in nature $\left({ }^{187} \mathrm{Re}\right.$ and ${ }^{3} \mathrm{H}$ with 2.5 and $18.6 \mathrm{keV}$ respectively) it is however difficult to obtain sensitivities much better than $\sim 1$ $\mathrm{eV}$. These experiments can therefore probe only quasi-degenerate neutrino masses (direct hierarchy). They represent however the only model independent approach to neutrino masses and are still therefore of key importance. $\beta \beta[0 \nu]$ experiments assume in fact the Majorana nature of the neutrino while cosmological observations depend on a number of astrophysical assumptions. Moreover, the projected sensitivities are in the range of the ${ }^{76} \mathrm{Ge} \beta \beta[0 \nu]$ claim, implying a substantial discovery potential of the upcoming experiments in case this claim would be confirmed.

So far, the best results have been obtained with magnetic spectrometers (MAINZ 10] and TROITSK [1]) which have reached sensitivities of the order of $2 \mathrm{eV}$. KATRIN [12], the only 
tritium experiment presently foreseen, has been discussed in the general talk of $\mathrm{C}$. Weinheimer. Its projected sensitivity is $0.2 \mathrm{eV}$ and will represent probably the end of the ${ }^{3} \mathrm{H}$ program. The KATRIN setup preparation is continuing without relevant delays and data taking should reasonably start in 2009.

Bolometric techniques offer a true calorimetric approach characterized by completely different (and probably safer) systematic uncertainties. A general presentation on the subject was given by Monica Sisti. Present sensitivities are however in the range of few eV 1314] while the proposed large scale extensions (MARE [15]) should dare to reach the $0.1 \mathrm{eV}$ region in the forthcoming decade. On the other hand, would this approach be successful, its extension would be strightforward.

\section{Cosmology}

Cosmological observations have started to provide valuable upper limits on absolute neutrino masses (see, e.g., the reviews [1617]), competitive with those from laboratory experiments. In particular, the combined analysis of high-precision data from Cosmic Microwave Background (CMB) anisotropies and Large Scale Structures (LSS) has already reached a sensitivity of $O(\mathrm{eV})$ (see, e.g., $\left[\begin{array}{l|l|l}18 & 19 & 20\end{array}\right)$ for the sum of the neutrino masses $\Sigma$,

$\Sigma=m_{1}+m_{2}+m_{3}$.

We recall that the total neutrino energy density in our Universe, $\Omega_{\nu} h^{2}$ (where $h$ is the Hubble constant normalized to $H_{0}=100 \mathrm{~km} \mathrm{~s}^{-1} \mathrm{Mpc}^{-1}$ ) is related to $\Sigma$ by the well-known relation $\Omega_{\nu} h^{2}=$ $\Sigma /(93.2 \mathrm{eV})$ [21], and plays an essential role in theories of structure formation. It can thus leave key signatures in LSS data (see, eg., 22]) and, to a lesser extent, in CMB data (see, e.g., 23]). Very recently, it has also been shown that accurate Lyman- $\alpha(\operatorname{Ly} \alpha)$ forest data 24], taken at face value, can improve the current $\mathrm{CMB}+\mathrm{LSS}$ constraints on $\Sigma$ by a factor of $\sim 3$, with important consequences on absolute neutrino mass scenarios $([252627])$.

In our session, the importance of cosmology for constraining neutrino masses and physics has been stressed in several talks. In particular, the most recent upper limits on neutrino masses from cosmology have been reported by Oystein Elgaroy (see [28]). Those limits now reach the impressive sub-eV level, which is competitive with future terrestrial neutrino experiments. Elgaroy however pointed out some of the caveats that should be borne in mind when interpreting the significance of these limits.

Recent high redshift matter clustering data derived from observations of Lyman- $\alpha$ forest clouds, have been extremely powerful in constraining neutrino masses. In his talk, Matteo Viel, explained in detail this new and promising cosmological tool. Lyman- $\alpha$ forest data allow to constrain the matter power spectrum from small scales of about $1 \mathrm{Mpc} / \mathrm{h}$ all the way to the horizon scale. The long lever arm and complementarity provided by such an analysis has previously led to a significant tightening of the constraints on the shape and the amplitude of the power spectrum of primordial density fluctuations. Viel then presented a combined analysis of the WMAP three year results with Lyman- $\alpha$ forest data (see [31]).

Gianpiero Mangano, presented new results from a recent analysis where the cosmological effects of interactions of neutrinos with cold Dark Matter (DM) is investigated 29]. This interaction produces diffusion-damped oscillations in the matter power spectrum, analogous to the acoustic oscillations in the baryon-photon fluid. It is therefore possible to obtain new and independent bounds from galaxy surveys like the recent Sloan Digital Sky Survey on the corresponding opacity defined as the ratio of neutrino-DM scattering cross section over DM mass. Those constraints have been then compared with the constraint from observation of neutrinos from supernova 1987A.

Paolo Serra, on the other hand, presented new results on the possible connection between neutrino physics and dark energy. In a recent analysis [30], by combining the ${ }^{76} \mathrm{Ge} \beta \beta[0 \nu]$ result with the WMAP 3-years data and other cosmological datasets a constraint on the dark energy equation of state of $-1.67<w<-1.05$ has been obtained at $95 \%$ c.l., ruling out a cosmological constant. Interestingly enough, coupled neutrino-dark energy models may be consistent 
with such equation of state. While future data are certainly needed for a confirmation of the controversial Heildelberg-Moscow claim, our result shows that future laboratory searches for neutrino masses may play a crucial role in the determination of the dark energy properties.

Properties of neutrinos, the lightest of all elementary particles, may be the origin of the entire matter-antimatter asymmetry of the universe. This requires that neutrinos are Majorana particles, which are equal to their antiparticles, and that their masses are sufficiently small. Pasquale di Bari, in his talk, showed that leptogenesis, the theory explaining the cosmic matter-antimatter asymmetry, predicts that all neutrino masses are smaller than $0.2 \mathrm{eV}$, which will be tested by forthcoming laboratory experiments and by cosmology (see 33]).

Francesco Villante presented how nucleosynthesis restrictions on mixing of active neutrinos with possible sterile ones are obtained with the account of experimentally determined mixing between all active neutrinos (see 34). The earlier derived bounds, valid in the absence of activeactive mixing, have been reanalyzed and significant difference is found in the resonance case. The results are obtained both analytically and numerically by solution of complete system of integro-differential kinetic equations. A good agreement between analytical and numerical approaches seems demonstrated.

Finally Tositaka Kajino (see 32]) reminded us that neutrino oscillations affect light element synthesis through the neutrino-process in supernova explosions. The ${ }^{7} \mathrm{Li}$ and ${ }^{11} \mathrm{~B}$ produced in a supernova explosion of a 16.2 solar-mass star model increase by factors of 1.9 and 1.3 in the case of large mixing angle solution with normal mass hierarchy and $\sin ^{2} 2 \theta_{13}>0.002$ compared with those without the oscillations. In the case of inverted mass hierarchy or nonadiabatic 13-mixing resonance, the increment of their yields is much smaller. Neutrino oscillations raise the reaction rates of charged-current neutrino-process reactions in the region outside oxygen-rich layers. The number ratio of ${ }^{7} \mathrm{Li} /{ }^{11} \mathrm{~B}$ could be a tracer of normal mass hierarchy and relatively large $\theta_{13}$, still satisfying $\sin ^{2} 2 \theta_{13}<0.1$, through future precise observa- tions in stars having strong supernova component.

\section{Conclusions}

Neutrino nature and masses represent key items for neutrino Physics in the next years. Some of the most powerful tools to scrutinize this sector, cosmology, $\beta \beta[0 \nu]$ and $\beta$ decay, were discussed in the session. Cosmological bounds and $\beta \beta[0 \nu]$ measurements offer the best sensitivities but strongly depend on to some extent untested assumptions the first and on nuclear matrix elements uncertainties the latter. Efforts to improve $\beta \beta[0 \nu]$ nuclear matrix elements reliability are in progress and their interplay with experimental activities will be crucial in the next future. On the other hand the rich program of experimental activities presented in the session is approaching the phase of production of results. Current experiments are probing the quasi-degenerate mass region, while a substantial coverage of the "inverted hierarchy" region would be achieved in the next generation $\beta \beta[0 \nu]$ experiments. These experiments will accommodate $\mathrm{O}$ (ton) of double beta decay emitters and will require improvements on background reduction and isotopes production. Concerning the quasi-degenerate mass region, the latest WMAP results seem to not leave much room for direct mass measurements with 200-meV sensitivity. Moreover, cosmological sensitivity is going to further improve with results from the Planck satellite mission. However, despite the impressive success of precision cosmology, cosmological bounds are derived within a system of assumptions and interpretations. Considering the importance of the neutrino mass question, and the difficulty in associating the cosmological limit to a precise systematic confidence level, direct measurements should be pursued up to their eventual technological limits. Probing this region is also crucial to verify the claim on $\beta \beta[0 \nu]$ of ${ }^{76} \mathrm{Ge}$, pointing to $\left|\left\langle m_{e e}\right\rangle\right|$ value around $0.4 \mathrm{eV}$.

In few words, several topics have been presented at our session. All of them extremely stimulating and worthwile of future developments and investigations. It will be duty of future experi- 
ment, on earth, in space or "in the universe" to scrutinize all these important hypotheses.

Acknowledgements We would like to thank the Organizers of the NOW-2006 workshop.

\section{REFERENCES}

1. O. Cremonesi, "Double Beta Decay: experiment and theory" in "Lepton and Photon 2005", World Scientific (2006) 310-323.

2. H.V. Klapdor-Kleingrothaus et al., Nucl. Instr. and Meth. A 510, 281 (2003); H.V. Klapdor-Kleingrothaus et al., Phys. Lett. B 586, 198 (2004)

3. C. Arnaboldi et al., Phys. Lett. B 584, 260 (2004); C. Arnaboldi et al., Phys. Rev. Lett. 95142501 (2005);

4. R. Arnold et al. (NEMO), Phys. Rev. Lett. 95, 182302 (2005) (arXiv:hep-ex/0507083); R. Arnold et al., Nucl. Phys. A 765, 483 (2006) (arXiv:hep-ex/0601021).

5. C.E. Aalseth et al., Nucl. Phys. B (Proc. Suppl.) 138217 (2005); arXiv:nucl-ex/0311013

6. M. Danilov et al., Phys. Lett. B 480, 12 (2000); D. Akimov et al., Nucl. Phys. B (Proc. Suppl.) 138, 224 (2005)

7. R. Ardito et al., arXiv:hep-ex/0501010 C. Arnaboldi et al. Nucl. Instr. and Meth. A 518, 775 (2004).

8. I. Abt et al., arXiv:hep-ex/0404039.

9. A.S. Barabash et al., arXiv:hep-ex/0602011.

10. C. Krauss et al., Eur. Phys. J. C 40, 447 (2005).

11. A.I. Belesev et al., Phys. Lett. B 350, 263 (1995); V.M. Lobashev et al., Phys. Lett. B 460, 227 (1999).

12. A. Osipowicz et al., arXiv:hep-ex/0109033 V.M. Lobashev, Nucl.Phys. A 719, 153 (2003).

13. C. Arnaboldi et al., Phys. Rev. Lett. 91, 161802 (2003); M. Sisti et al, NIM A 520, 125 (2004).

14. M. Galeazzi et al., Phys. Rev. C 63, 014302 (2001);

15. C.

Arnaboldiet al., http://crio.mib.infn.it/wig/silicini/proposal/; A. Monfardini et al, arXiv:hep-ex/0509038
16. V. Barger, D. Marfatia and K. Whisnant, Int. J. Mod. Phys. E 12, 569 (2003).

17. A.D. Dolgov, Phys. Rept. 370, 333 (2002).

18. WMAP Collaboration, C.L. Bennett et al., Astrophys. J. Suppl. 148, 1 (2003).

19. SDSS Collaboration, M. Tegmark et al., Phys. Rev. D 69, 103501 (2004).

20. See also O. Lahav, "Massive Neutrinos and Cosmology," in Neutrino 2004 [?].

21. Review of Particle Physics, S. Eidelman et al., Phys. Lett. B 592, 1 (2004).

22. W. Hu, D.J. Eisenstein, and M. Tegmark, Phys. Rev. Lett. 80, 5255 (1998).

23. C.P. Ma and E. Bertschinger, Astrophys. J. 455, 7 (1995).

24. SDSS Collaboration, P. McDonald et al., astro-ph/0405013

25. U. Seljak et al., astro-ph/0407372.

26. G. L. Fogli, E. Lisi, A. Marrone, A. Melchiorri, A. Palazzo, P. Serra and J. Silk, Phys. Rev. D 70 (2004) 113003 arXiv:hep-ph/0408045.

27. G. L. Fogli et al., arXiv:hep-ph/0608060.

28. J. R. Kristiansen, O. Elgaroy and H. Dahle, arXiv:astro-ph/0611761.

29. G. Mangano, A. Melchiorri, P. Serra, A. Cooray and M. Kamionkowski, $\begin{array}{llllll}\text { Phys. Rev. D } & \mathbf{7 4} & \text { (2006) } & 043517\end{array}$ arXiv:astro-ph/0606190].

30. A. De La Macorra, A. Melchiorri, P. Serra and R. Bean, arXiv:astro-ph/0608351.

31. M. Viel, M. G. Haehnelt and A. Lewis, Mon. Not. Roy. Astron. Soc. Lett. 370 (2006) L51 arXiv:astro-ph/0604310.

32. T. Yoshida, T. Kajino, H. Yokomakura, K. Kimura, A. Takamura and D. H. Hartmann, arXiv:astro-ph/0606042.

33. S. Blanchet and P. Di Bari, JCAP 0606 (2006) 023 arXiv:hep-ph/0603107.

34. A. D. Dolgov and F. L. Villante, Nucl. Phys. B 679 (2004) 261 arXiv:hep-ph/0308083. 